\title{
Randomized Phase II Study of Afatinib Plus Simvastatin Versus Afatinib Alone in Previously Treated Patients with Advanced Nonadenocarcinomatous Non-small Cell Lung Cancer
}

\author{
Youngjoo Lee, MD, PhD' \\ Ki Hyeong Lee, MD, PhD² \\ Geon Kook Lee, MD, PhD ${ }^{1}$ \\ Soo-Hyun Lee, MD \\ Kun Young Lim, MD \\ Jungnam Joo, $\mathrm{PhD}^{3}$ \\ Yun Jung Go, MS \\ Jin Soo Lee, MD, PhD ${ }^{1}$ \\ Ji-Youn Han, MD, PhD ${ }^{1}$
}

${ }^{1}$ Center for Lung Cancer,

Research Institute and Hospital,

National Cancer Center, Goyang,

${ }^{2}$ Department of Hematology-Oncology,

Chungbuk National University Hospital,

Cheongju, ${ }^{3}$ Biometric Research Branch,

Research Institute and Hospital, National

Cancer Center, Goyang, Korea

Correspondence: Ji-Youn Han, MD, PhD

Center for Lung Cancer, Research Institute and

Hospital, National Cancer Center, 323 Ilsan-ro,

Ilsandong-gu, Goyang 10408, Korea

Tel: 82-31-920-1154

Fax: 82-31-920-2587

E-mail: jymama@ncc.re.kr

Received November 18, 2016

Accepted December 30, 2016

Published Online January 13, 2017

\begin{abstract}
Purpose
This phase II study examined whether the addition of simvastatin to afatinib provides a clinical benefit compared with afatinib monotherapy in previously treated patients with nonadenocarcinomatous non-small cell lung cancer (NA-NSCLC).
\end{abstract}

\section{Materials and Methods}

Patients with advanced NA-NSCLC who progressed after one or two chemotherapy regimens were randomly assigned to a simvastatin ( $40 \mathrm{mg} /$ day) plus afatinib (40 mg/day) (AS) arm or to an afatinib (A) arm. The primary endpoint was response rate (RR).

\section{Results}

Sixty-eight patients were enrolled (36 in the AS arm and 32 in the A arm). The RR was 5.7\% (95\% confidence interval [Cl], 0.7 to 19.2) for AS and 9.4\% (95\% Cl, 2.0 to 25.0$)$ for A $(p=0.440)$. In arms AS and A, the median progression-free survival (PFS) was 1.0 versus 3.6 months $(p=0.240)$ and the overall survival was 10.0 months versus 7.0 months $(p=0.930)$, respectively. Skin rash, stomatitis, and diarrhea were the most common adverse events in both arms. More grade 3 or 4 diarrhea was observed in arm A (18.8\% vs. $5.6 \%$ in arm AS). In all patients, the median PFS for treatment including afatinib was not correlated with the status of epidermal growth factor receptor (EGFR) mutation $(p=0.122)$, EGFR fluorescence in situ hybridization ( $p=0.944)$, or EGFR immunohistochemistry $(p=0.976)$. However, skin rash severity was significantly related to the risk of progression for afatinib (hazard ratio for skin rash grade $\geq 2$ vs. grade $<2,0.44 ; 95 \% \mathrm{Cl}, 0.25$ to $0.78 ; \mathrm{p}=0.005$ ).

\section{Conclusion}

There were no significant differences in the efficacy between AS and A arms in patients with NA-NSCLC.

\section{Introduction}

The molecularly-targeted drugs that inhibit epidermal growth factor receptor (EGFR), gefitinib and erlotinib, were initially expected to treat the vast majority of non-small cell lung cancer (NSCLC) because overexpression of EGFR protein is frequently detected in this type of tumor [1]. However, many preclinical and clinical studies have demonstrated that these drugs are only effective in a specific subset of NSCLC,
Key words

EGFR tyrosine kinase inhibitor, EGFR mutation, Non-small-cell lung carcinoma, Squamous cell carcinoma, Statin 
NSCLC patients, derive limited clinical benefits from EGFRTKIs. In a meta-analysis study analyzing 11 randomized controlled studies, including 1,605 patients with advanced WT EGFR NSCLC, first-generation EGFR-TKIs showed a significantly lower RR of 7.2\% and a higher risk of progression of 1.41 when compared with conventional chemotherapy in patients with WT EGFR NSCLC [9]. Although one EGFRTKI, erlotinib, was approved in patients with WT EGFR NSCLC, the survival benefit was modest in the BR.21 trial comparing this drug with a placebo in an unselected pretreated NSCLC population. Specifically, the improvement in median PFS was 0.4 months and the improvement in median overall survival (OS) was 2.0 months [10]. Thus, there are major unmet needs in using EGFR-TKIs to treat patients with advanced NSCLC with WT EGFR.

We previously conducted a randomized phase II clinical study to improve gefitinib efficacy by adding simvastatin in unselected advanced NSCLC patients [11]. One of the main reasons for primary resistance to EGFR-TKIs is that there are alternative mechanisms for persistent activation of EGFR downstream signaling, including both the RAS/ERK and phosphoinositide 3-kinase (PI3K) / Akt kinase pathways [12]. Thus, we hypothesized that simultaneous inhibition of both pathways would inhibit tumor cell survival more effectively in tumors resistant to EGFR-TKIs. One candidate drug combination is EGFR-TKIs and statins, which are irreversible inhibitors of 3-hydroxy-3-methylglutaryl-coenzyme A reductase that are used to treat hypercholesterolemia by blocking the mevalonate biosynthesis pathway [13]. Isoprenoids, which are products of the mevalonate pathway, are attached to RAS proteins to allow them to anchor in the cell membrane, where they perform biological roles [14,15]. By interrupting the biosynthesis of mevalonate, statins may inhibit activation of RAS and downstream signaling cascades, including the RAF/MEK/ERK and PI3K/AKT cascades, which play critical roles in regulating cell survival and proliferation. Thus, this may be a promising therapeutic approach to overcoming tumor resistance to EGFR-TKIs. In our previous study, the combination of gefitinib and simvastatin showed higher RR ( $39 \%$ vs. $8 \%, \mathrm{p}=0.06)$ than gefitinib alone in the subgroup of nonadenocarcinomatous (NA)NSCLC, which is unlikely to have EGFR mutations. This finding suggests that simvastatin may enhance sensitivity to gefitinib in NA-NSCLC that is resistant to gefitinib. Moreover, several preclinical studies demonstrated that combined gefitinib and lovastatin exerted significant synergic cytotoxic effects in vitro in squamous cell carcinomas, NSCLC, and colon carcinoma cell lines that do not possess the activating EGFR mutations [16,17].

Simvastatin is metabolized in liver cells by CYP3A4, and its concurrent use with other substrates of this enzyme is contraindicated because of increased toxicity [18]. Thus, the com- bination of simvastatin and gefitinib or erlotinib would not be suitable for a clinical study because they are also CYP3A4 substrates [19]. However, afatinib, a second-generation irreversible EGFR-TKI that is not a CYP3A4 substrate, was identified as a good candidate for combination with simvastatin. Therefore, we conducted a randomized phase II study to compare the efficacy of afatinib and simvastatin (AS) with afatinib alone (A) in previously treated patients with NA-NSCLC.

\section{Materials and Methods}

\section{Patients}

Eligible patients were $\geq 18$ years old with pathologically confirmed stage IIIB/IV NA-NSCLC (e.g., squamous cell or large cell carcinoma) that progressed after first- or secondline cytotoxic chemotherapy regimens, including at least one platinum-containing regimen. Patients with an Eastern Cooperative Oncology Group (ECOG) performance status (PS) of 0,1 , and 2 and adequate organ function were eligible. Patients had a measurable lesion according to the Response Evaluation Criteria in Solid Tumors (RECIST) ver. 1.1 [20]. Patients receiving prior treatment with small molecules or antibodies that inhibit EGFR (e.g., gefitinib, erlotinib, and cetuximab) were excluded.

All patients provided written informed consent, and this study was approved by the Institutional Review Board. The study was conducted in accordance with the Declaration of Helsinki and the International Conference on Harmonization/Good Clinical Practice. This study is registered with ClinicalTrials.gov under identifier NCT01156545.

\section{Study design and treatment}

This was a multicenter, open, randomized, phase II study to evaluate the synergistic effects of afatinib plus simvastatin in pretreated NA-NSCLC patients. The stratification factors for randomization were ECOG PS (0 vs. 1 or 2).

After allocation to the treatment arms, patients received continuous daily treatment with either afatinib plus simvastatin or afatinib plus the best supportive care until disease progression, unacceptable adverse events, or another reason necessitating withdrawal. The treatments were administered as 28-day courses. The starting doses of AS were $40 \mathrm{mg}$ once daily. Dose escalation of afatinib to $50 \mathrm{mg}$ was permitted for individual patients after one cycle if there were minimal adverse events at $40 \mathrm{mg}$. When drug-related adverse events occurred, the afatinib dose was reduced in increments of 
$10 \mathrm{mg}$, with the lowest dose being $20 \mathrm{mg}$. There was no dose reduction for simvastatin.

\section{Tumor assessment}

The tumor response was assessed after 4, 8, 16, and 24 weeks and every 8 weeks thereafter until progression or withdrawal for another reason. After week 48, the response was assessed every 12 weeks. Tumor response and progression were assessed using RECIST ver. 1.1 [20].

\section{Statistical analyses}

The primary endpoint was the objective response, as determined according to RECIST ver. 1.1 [20]. The objective response rate (ORR) was calculated as the ratio of the number of responders to the number of patients assessed for tumor response. Responders were defined as patients showing complete response (CR) or partial response (PR). We expected that afatinib plus simvastatin would increase ORR by $35 \%$ from $10 \%$ for afatinib alone based on the results of Han et al. [11]. Including 62 patients (31 in each arm) would give statistical power of $80 \%$ by Fisher exact test (one-sided, $\alpha=0.15)$. Considering a $10 \%$ attrition rate, we planned to recruit a total of 68 patients (34 in each arm).

The secondary endpoints were PFS, OS, and adverse events. Adverse events were graded according to the Com- mon Terminology Criteria for Adverse Events ver. 4.0. PFS was calculated as the duration from the date of randomization to the date of disease progression or death, whichever occurred first. OS was calculated as the duration from the date of randomization to the date of death. Survival time was estimated using the Kaplan-Meier method, and the survival difference between groups was assessed using the log-rank test. $\mathrm{p}$-values were one-sided. A p $<0.025$ was considered to be significant.

\section{Exploratory biomarker study}

Archival tumor tissues were collected before treatment in all patients. We checked the EGFR status by three methods: mutation status by direct sequencing, gene copy number by fluorescence in situ hybridization (FISH), and protein expression by immunohistochemistry (IHC) staining.

Nucleotide sequencing of the kinase domain of the EGFR gene (exons 19, 20, and 21) was performed by direct sequencing of the individual exons. The details describing the sequencing procedure have been described elsewhere [21]. The EGFR gene copy number was calculated with the FISH test using the LSI EGFR SpectrumOrange/CEP 7 SpectrumGreen Probe (Vysis, Abbott Molecular, Des Plaines, IL) according to the standard protocol [22]. Tumors were considered to have an increased copy number (FISH-positive) if they showed gene amplification or chromosome 7 high

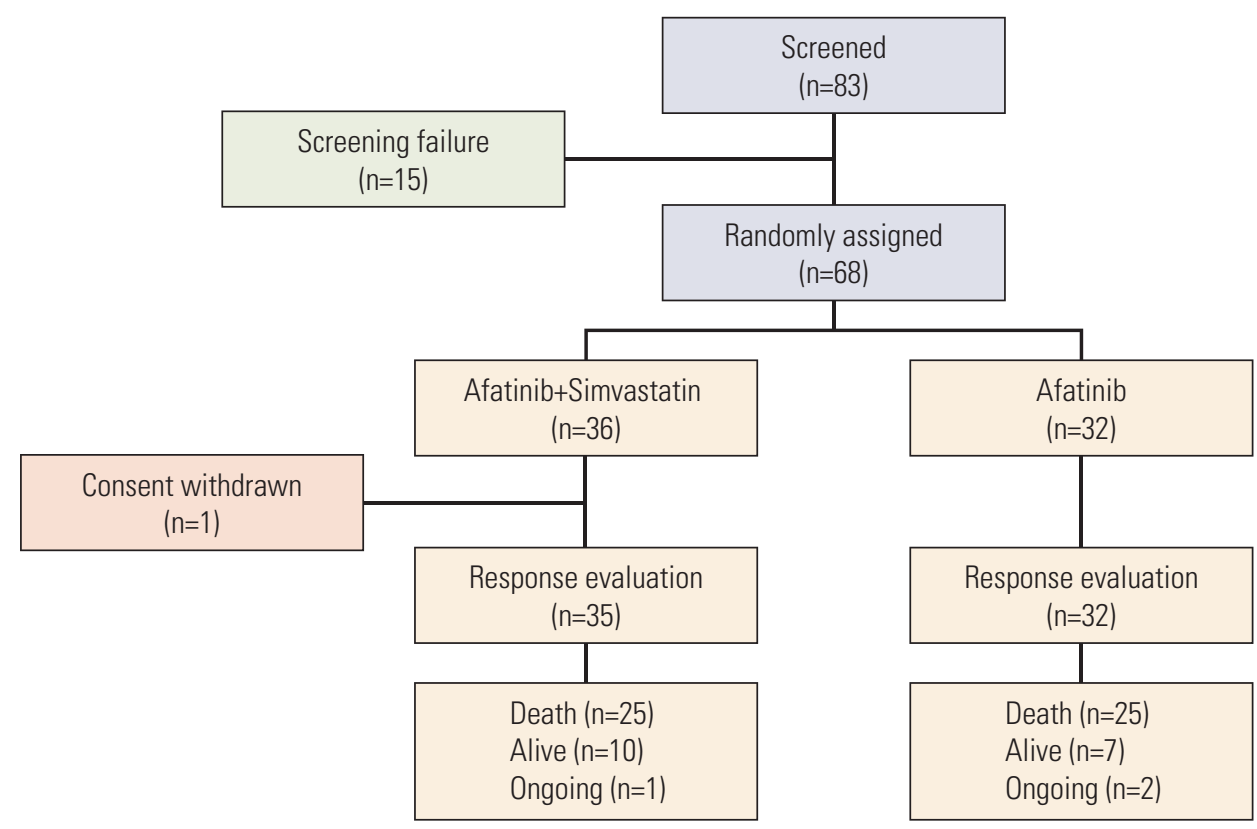

Fig. 1. CONSORT diagram. 
Table 1. Patient characteristics

\begin{tabular}{|c|c|c|c|}
\hline Characteristic & Afatinib+Simvastatin & Afatinib & p-value \\
\hline Age, median (range, yr) & $59(44-80)$ & $67(44-78)$ & 0.008 \\
\hline \multicolumn{4}{|l|}{ Sex } \\
\hline Male & $32(88.9)$ & $27(84.4)$ & 0.725 \\
\hline Female & $4(11.1)$ & $5(15.6)$ & \\
\hline \multicolumn{4}{|l|}{ Smoking } \\
\hline Never & $4(11.1)$ & $1(3.1)$ & 0.460 \\
\hline Ever & $31(86.1)$ & $30(93.8)$ & \\
\hline Unknown & $1(2.8)$ & $1(3.1)$ & \\
\hline \multicolumn{4}{|l|}{ Pathology } \\
\hline Squamous cell carcinoma & $33(91.7)$ & $31(96.9)$ & 0.616 \\
\hline Other & $3(8.3)$ & $1(3.1)$ & \\
\hline \multicolumn{4}{|l|}{ ECOG PS } \\
\hline $0-1$ & $27(75.0)$ & $24(75.0)$ & 0.854 \\
\hline 2 & $9(25.0)$ & $8(25.0)$ & \\
\hline \multicolumn{4}{|l|}{ No. of prior chemotherapies } \\
\hline 1 & $21(58.3)$ & $19(59.4)$ & 1.000 \\
\hline 2 & $15(41.7)$ & $13(40.6)$ & \\
\hline \multicolumn{4}{|l|}{ EGFR mutation } \\
\hline Wild type & $32(88.8)$ & $28(87.6)$ & 1.000 \\
\hline Mutant type ${ }^{a)}$ & $2(5.6)$ & $2(6.2)$ & \\
\hline Unknown & $2(5.6)$ & $2(6.2)$ & \\
\hline \multicolumn{4}{|l|}{ EGFR FISH } \\
\hline Negative & $30(83.4)$ & $26(81.3)$ & 1.000 \\
\hline Positive & $3(8.3)$ & $2(6.2)$ & \\
\hline Unknown & $3(8.3)$ & $4(12.5)$ & \\
\hline \multicolumn{4}{|l|}{ EGFR IHC } \\
\hline Negative & $14(38.9)$ & $10(31.3)$ & 0.698 \\
\hline Positive & $17(47.2)$ & $15(46.9)$ & \\
\hline Unknown & $5(13.9)$ & 7 (21.9) & \\
\hline
\end{tabular}

ECOG, Eastern Cooperative Oncology Group; PS, performance status; EGFR, epidermal growth factor receptor; FISH, fluo-

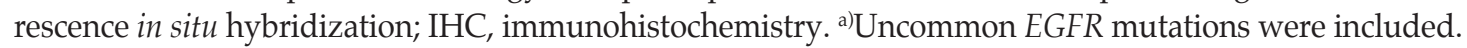

Table 2. Overall response rate

\begin{tabular}{|c|c|c|c|}
\hline Response & Afatinib+Simvastatin & Afatinib & p-value \\
\hline Complete response & 0 & 1 & \\
\hline Partial response & 2 & 2 & \\
\hline Stable disease & 14 & 21 & \\
\hline Disease progression & 19 & 8 & \\
\hline Not evaluable & 1 & 0 & \\
\hline Overall response rate (complete response+partial response) $(\%)$ & 5.7 & 9.4 & 0.430 \\
\hline
\end{tabular}


polysomy based on previously described criteria [22]. The intensity of EGFR IHC staining (Zymed, Thermo Fisher Scientific, Fremont, CA) was scored as follows: 0, no membrane staining; $1+$, faint, partial membrane staining; $2+$, weak, complete membrane staining in $>10 \%$ of tumor cells; $3+$, intense complete membrane staining in $>10 \%$ of tumor cells. Those with a score of $2+$ or $3+$ were classified as IHC-positive.

\section{Results}

\section{Patient characteristics}

Between November 2012 and September 2015, 83 patients from two centers in Korea were screened and 68 patients were enrolled (Fig. 1). Thirty-six patients were allocated to the AS arm and 32 to the A arm. After stating the allocated treatment, six patients discontinued the study due to toxicity $(n=1)$ or patient refusal $(n=5)$ (one in the AS arm and five in the A arm). Patient characteristics are listed in Table 1. Most patients were male $(87 \%)$, ever-smokers $(92 \%)$, had squamous cell carcinoma histology (94\%), and good ECOG 0-1 performance $(75 \%)$. Other histological subtypes included sarcomatoid carcinoma $(n=1)$ and NSCLC-not otherwise speci- fied $(n=3)$. Demographic baseline characteristics, except age, were generally balanced between the two groups.

\section{Efficacy}

Sixty-seven patients were assessed for response evaluation. One patient showed a CR and four patients showed a PR. The ORR was 5.7\% (95\% confidence interval [CI], 0.7 to 18.7) for the AS arm and 9.4\% (95\% CI, 2.0 to 25.0) for the A arm $(\mathrm{p}=0.430)$ (Table 2).

The median follow-up time for all patients was 22.3 months. A total of 50 deaths $(73.5 \%)$ occurred during data analysis. There was no difference in median PFS between the AS group (1.0 month [95\% CI, 0.5 to 1.4]) and the A group (3.6 months [95\% CI, 3.0 to 4.1]) ( $\mathrm{p}=0.240)$ (Fig. 2A). The hazard ratio (HR) for progression was 1.38 (95\% CI, 0.84 to 2.29; $\mathrm{p}=0.898$ ). The median OS was 10.0 months (95\% CI, 6.4 to 13.8) for the AS arm and 7.0 months (95\% CI, 6.1 to 7.9) for the $A$ arm ( $p=0.930)$ (Fig. 2B). The HR for death was 1.03 (95\% CI, 0.58 to 1.80; $\mathrm{p}=0.466$ ). Subsequent chemotherapy after progression was given to 27 of the 35 patients $(77.1 \%)$ in the AS arm and 18 of the 28 patients $(64.3 \%)$ in the A arm $(\mathrm{p}=0.262)$.

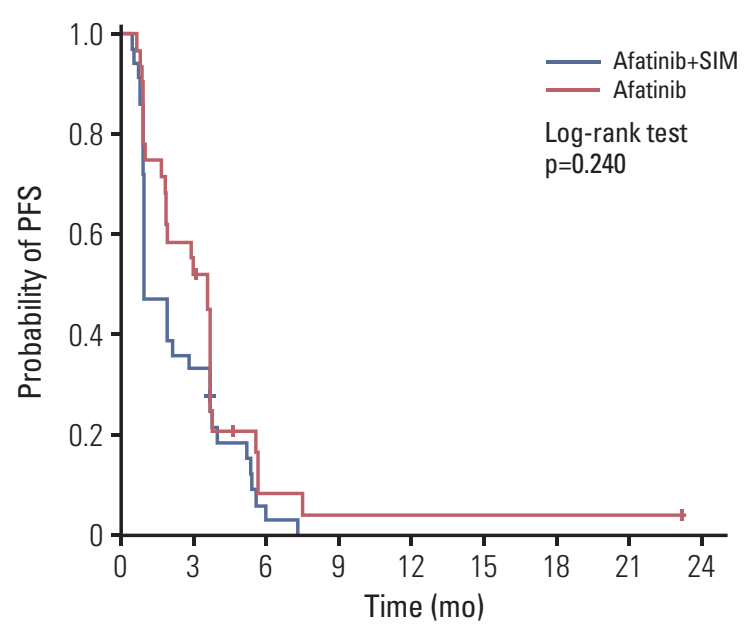

\begin{tabular}{lcc} 
Arm & Event & Median PFS (mo) \\
Afatinib+SIM (n=36) & 33 & 1.0 \\
Afatinib $(\mathrm{n}=32)$ & 25 & 3.6 \\
\hline
\end{tabular}

A

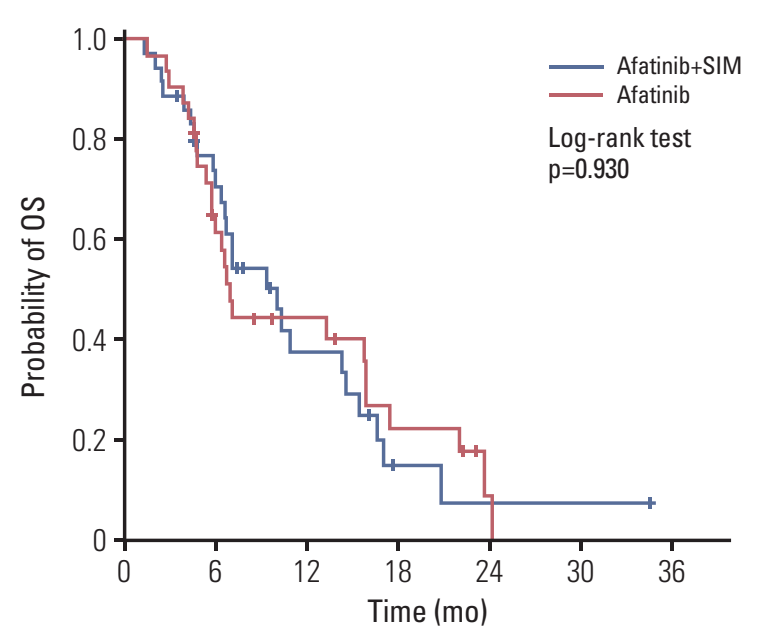

\section{Arm Event Median OS (mo)}

Afatinib+SIM (n=36) $\quad 25 \quad 10.0$

Afatinib (n=32) $\quad 25 \quad 7.0$

Fig. 2. Survival outcomes by treatment arm. (A) Progression-free survival (PFS). (B) Overall survival (OS). SIM, simvastatin. 
Table 3. Adverse effects

\begin{tabular}{|c|c|c|c|c|}
\hline \multirow{2}{*}{ Adverse effect } & \multicolumn{2}{|c|}{ Afatinib+Simvastatin } & \multicolumn{2}{|c|}{ Afatinib } \\
\hline & $\geq$ Grade 3 & Total grade & $\geq$ Grade 3 & Total grade \\
\hline AST/ ALT increased & $1(2.8)$ & $4(11.1)$ & 0 & $1(3.1)$ \\
\hline Creatinine increased & 0 & $4(11.1)$ & $1(3.1)$ & $7(21.9)$ \\
\hline QT prolonged & 0 & $6(16.7)$ & $1(3.1)$ & $4(12.5)$ \\
\hline Fatigue & 0 & $10(27.8)$ & $1(3.1)$ & $11(34.4)$ \\
\hline Anorexia & $1(2.8)$ & $11(30.6)$ & 0 & $14(43.8)$ \\
\hline Weight loss & 0 & $2(5.6)$ & 0 & $6(18.8)$ \\
\hline Diarrhea & $2(5.6)$ & $23(63.9)$ & $6(18.8)$ & $27(84.4)$ \\
\hline Stomatitis & 0 & $24(66.7)$ & $2(6.3)$ & $25(78.1)$ \\
\hline Dry skin & 0 & $7(19.4)$ & 0 & $6(18.8)$ \\
\hline Pruritus & 0 & $15(41.7)$ & 0 & $11(34.4)$ \\
\hline Skin rash & $1(2.8)$ & $28(77.8)$ & $1(3.1)$ & $25(78.1)$ \\
\hline Paronychia & 0 & $4(11.1)$ & 0 & $4(12.5)$ \\
\hline Hand-foot syndrome & 0 & $5(13.9)$ & 0 & $5(15.6)$ \\
\hline Esophageal ulcer & $1(2.8)$ & $1(2.8)$ & 0 & 0 \\
\hline Idiopathic bilateral vocal cord palsy & 0 & 0 & $1(3.1)$ & $1(3.1)$ \\
\hline
\end{tabular}

AST, aspartate aminotransferase; ALT, alanine transaminase.

Table 4. EGFR biomarker and treatment efficacy

\begin{tabular}{|c|c|c|c|c|c|}
\hline EGFR biomarker & No. & $\begin{array}{l}\text { Pathology } \\
\text { SCC }\end{array}$ & $\begin{array}{c}\text { Smoking } \\
\text { never }\end{array}$ & $\begin{array}{c}\text { Response } \\
\mathrm{CR}+\mathrm{PR}\end{array}$ & $\begin{array}{l}\text { PFS } \\
\text { (mo) }\end{array}$ \\
\hline EGFR wild type & 60 & $56(93)$ & $4(7)$ & $3(5)$ & 1.9 \\
\hline EGFR mutation type & 4 & $4(100)$ & 0 & $2(50)$ & 2.9 \\
\hline Exon 19 deletion & 1 & SCC & Ex-smoker & CR & 23.2 \\
\hline Exon 20 G810S & 1 & SCC & Ex-smoker & PR & 2.9 \\
\hline Exon $20 \mathrm{~V} 786 \mathrm{M}$ & 1 & SCC & Ex-smoker & $\mathrm{PD}$ & 1.0 \\
\hline Exon 21 G863S & 1 & SCC & Ex-smoker & SD & 5.2 \\
\hline EGFR/FISH negativity & 56 & $54(96)$ & $3(5)$ & $3(5)$ & 1.9 \\
\hline EGFR/FISH positivity & 5 & $4(80)$ & $1(20)$ & $2(40)$ & 3.6 \\
\hline High polysomy & 1 & SCC & Current smoker & PR & 5.4 \\
\hline High polysomy & 1 & SCC & Ex-smoker & SD & 4.0 \\
\hline Gene amplification & 1 & NSCLC-NOS & Never smoker & PR & 3.6 \\
\hline Botha) & 1 & $\mathrm{SCC}$ & Current smoker & PD & 1.0 \\
\hline Botha) & 1 & SCC & Ex-smoker & PD & 0.9 \\
\hline EGFR/IHC negativity & 24 & $21(88)$ & $4(17)$ & $1(4)$ & 3.6 \\
\hline EGFR/IHC positivity & 32 & $32(100)$ & $1(3)$ & $4(12.5)$ & 1.9 \\
\hline $2+$ & 16 & $16(100)$ & 0 & $1(6)$ & 1.0 \\
\hline $3+$ & 16 & $16(100)$ & $1(6)$ & $3(19)$ & 1.9 \\
\hline
\end{tabular}

Values are presented as number (\%). EGFR, epidermal growth factor receptor; SCC, squamous cell carcinoma; CR, complete response; PR, partial response; PFS, progression-free survival; FISH, fluorescence in situ hybridization; IHC, immunohisto-

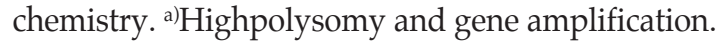




\section{Safety}

No patient in either arm received an escalated dose of afatinib of $50 \mathrm{mg} /$ day. The dose of afatinib was reduced to 30 $\mathrm{mg} /$ day in 14 patients $(20.6 \%$ ) (six in the AS arm and eight in the A arm), whereas the dose was reduced to $20 \mathrm{mg} /$ day in one patient in the AS arm. The main reasons for the dose reduction were stomatitis and skin rash. Five patients $(7.4 \%)$ stopped treatment because of adverse events (two in the AS arm and three in the A arm). The mean relative dose intensities of the AS and A arms were $95.9 \%$ and $94.4 \%$, respectively $(p=0.667)$. The safety profiles of both treatment arms were similar (Table 3). There were no treatment-related mortalities in either arm. Common adverse events included skin rash (77.8\% in the AS arm vs. $78.1 \%$ in the A arm), diarrhea (63.9\% vs. $84.4 \%$ ), and stomatitis ( $66.7 \%$ vs. $78.1 \%)$. Interestingly, more patients experienced grade 3 or 4 diarrhea in the $\mathrm{A}$ arm $(5.6 \%$ vs. $18.8 \%)$. Close relationships between common adverse effects were observed. Patients experiencing grade $\geq 2$ skin rash had a higher incidence of grade $\geq 2$ stomatitis or diarrhea than those with grade $<2$ skin rash (grade $\geq 2$ stomatitis, $56.0 \%$ vs. $27.9 \%, \mathrm{p}=0.022$; grade $\geq 2$ diarrhea, $48.0 \%$ vs. $27.9 \%, \mathrm{p}=0.095)$. Additionally, there was a significant association between grade $\geq 2$ stomatitis and grade $\geq 2$ diarrhea $(\mathrm{p}=0.046)$.

\section{EGFR status and clinical outcome}

Among a total of 68 patients, the tumors of 64 patients were available for EGFR mutation analysis, 61 for the FISH test, and 56 for IHC staining analysis. EGFR mutations were detected in four of 64 cases $(6.3 \%$ ) (one exon 19 deletion, one exon 20 G810S, one exon 20 V786M, and one exon 21 G863S) (Table 4). Of the 61 tumors suitable for EGFR/FISH analysis, five $(8.2 \%$ ) were FISH-positive (two high polysomy, one gene amplification, and two both). Upon EGFR IHC analysis, positive expression was shown in 32 patients $(57 \%)$ (16 for $2+$ and 16 for $3+)$. There was no difference in the rate of EGFR mutation ( $5.6 \%$ vs. $6.2 \%$ ), FISH-positivity ( $8.3 \%$ vs. $6.2 \%$ ), or IHC-positivity ( $47.2 \%$ vs. $46.9 \%$ ) between arms. In addition, the rate of these biomarkers did not differ with age, sex, ECOG PS, pathology (squamous cell carcinoma vs. others), or smoking status. There was no overlap between EGFR mutation and FISH positivity.

Upon analysis of all patients, EGFR mutation and EGFR/ FISH status were significantly associated with the response to treatment, including afatinib, whereas EGFR IHC status was not. The EGFR-mutant patients showed higher ORR than the EGFR wild-type patients $(50.0 \%$ vs. $5.0 \%$, respectively; $\mathrm{p}=0.028$ ) (Table 4). In addition, the EGFR/FISH-positive patients showed higher ORR than the EGFR/FISHnegative patients $(40.0 \%$ vs. $5.4 \%$, respectively; $\mathrm{p}=0.049)$.
Thus, the EGFR-mutant or EGFR/FISH-positive patients $(n=9)$ showed significantly higher ORR than those with tumors harboring both WT EGFR and EGFR/FISH-negativity $(\mathrm{n}=52)(44.4 \%$ vs. $1.9 \%$, respectively; $\mathrm{p}=0.001)$. However, the EGFR mutation and EGFR/FISH status were not correlated with median PFS for treatment. The median PFS was 2.9 months (95\% CI, 0.1 to 6.2) in the EGFR-mutant patients vs. 1.9 months (95\% CI, 0.9 to 2.9 ) in the WT EGFR patients (HR, $0.42 ; 95 \% \mathrm{CI}, 0.13$ to $1.36 ; \mathrm{p}=0.122)$, and 3.6 months $(95 \%$ CI, 0.1 to 9.2) in the EGFR/FISH-positive patients versus 1.9 months (95\% CI, 0.9 to 2.9) in the EGFR/FISH-negative patients (HR, 0.97; 95\% CI, 0.38 to 2.45; $\mathrm{p}=0.944$ ). The EGFR IHC status was not related to median PFS (IHC-positive vs. IHC-negative; 1.9 months vs. 3.6 months; $\mathrm{p}=0.976$ ).

When the analysis was limited to 60 patients with tumors harboring WT EGFR, the ORR was $6.3 \%$ for the AS arm versus $3.6 \%$ for the $A$ arm $(p=1.000)$, median PFS was 1.0 versus 3.6 months ( $\mathrm{p}=0.218$ ), and median OS was 10.0 months versus 6.8 months $(\mathrm{p}=0.815)$. In the subgroup with $E G F R$-mutant tumors $(n=4)$, the ORR of the AS versus $A$ arm was $0.0 \%$ versus $100.0 \%(\mathrm{p}=0.333)$, the median PFS was 1.9 months versus 2.9 months ( $\mathrm{p}=0.433$ ), and the median OS was 6.7 months versus 7.1 months ( $\mathrm{p}=0.815)$. In terms of $E G F R$ FISH status, the negative subgroup $(n=56)$ had an ORR of $3.3 \%$ versus $7.7 \%$ (AS vs. $\mathrm{A}, \mathrm{p}=0.592$ ), a median PFS of 1.0 versus 3.6 months ( $\mathrm{p}=0.209$ ), and a median OS of 10.0 months versus 7.1 months $(\mathrm{p}=0.318)$, whereas the positive subgroup $(\mathrm{n}=5)$ had an ORR of $33.3 \%$ vs. $50.0 \%$ (AS vs. A, $\mathrm{p}=1.000$ ), a median PFS of 4.0 vs. 0.9 months ( $p=0.207)$, and a median OS of 6.0 months versus 4.9 months ( $\mathrm{p}=0.586$ ). The subgroup analysis with EGFR-IHC negative tumors $(\mathrm{n}=4)$ had an ORR of $0.0 \%$ vs. $10.0 \%$ (AS vs. A, $\mathrm{p}=0.417$ ), a median PFS of 1.0 months versus 3.6 months ( $\mathrm{p}=0.789$ ), and a median OS of 11.0 months versus 7.0 months $(\mathrm{p}=0.918)$, whereas the positive subgroup analysis had an ORR of $11.8 \%$ versus $13.3 \%$ (AS vs. A, $\mathrm{p}=1.000$ ), a median PFS of 1.0 versus 1.9 months $(\mathrm{p}=0.122)$, and a median OS of 7.1 months versus 13.3 months ( $\mathrm{p}=0.329$ ).

\section{Adverse effects as a predictive marker}

For all patients receiving afatinib, the adverse effects skin rash, stomatitis, and diarrhea were not significantly associated with the ORR, regardless of whether the patients received simvastatin. However, for the 16-week PFS rate, the grade of skin rash and stomatitis was significantly related to the efficacy of afatinib (Fig. 3). Patients who experienced grade $\geq 2$ skin rash or stomatitis showed a significantly higher 16-week PFS rate than those with skin rash or stomatitis of grade $<2$ (skin rash, $48.0 \%$ vs. $14.0 \%$, $\mathrm{p}=0.002$; stomatitis, $46.2 \%$ vs. $14.3 \%, \mathrm{p}=0.004)$. These results remained unchanged in the landmark analysis at 4 weeks (skin rash, $57.1 \%$ vs. $20.0 \%, \mathrm{p}=0.006$; stomatitis, $54.5 \%$ vs. $20.7 \%$, 


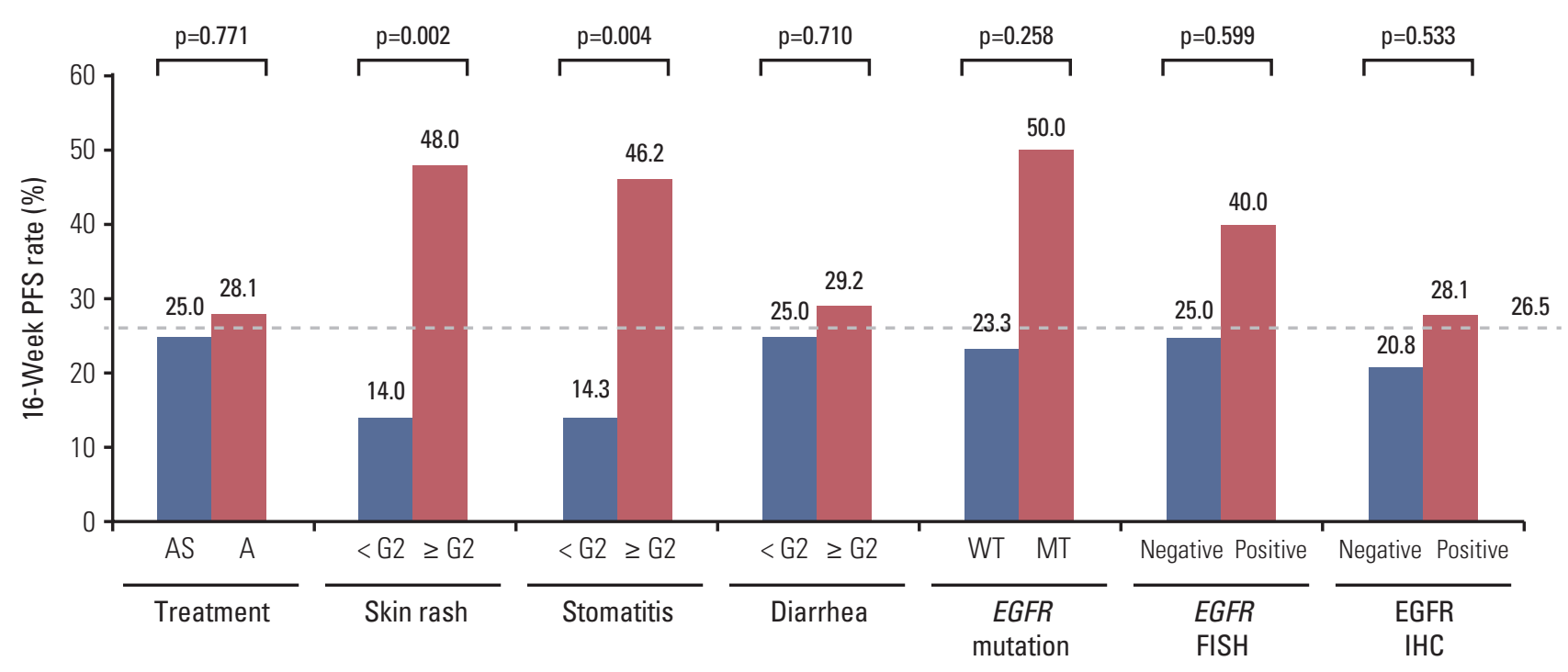

Fig. 3. Sixteen-week progression-free survival (PFS) rate by treatment arm, adverse effects, and epidermal growth factor receptor (EGFR) biomarkers. AS, simvastatin plus afatinib; A, afatinib; WT, wild type; MT, mutant; FISH, fluorescence in situ hybridization; IHC, immunohistochemistry.

Table 5. Progression-free survival analysis

\begin{tabular}{|c|c|c|c|c|}
\hline \multirow{2}{*}{ Variable } & \multicolumn{2}{|c|}{ Univariate } & \multicolumn{2}{|c|}{ Multivariate } \\
\hline & HR $(95 \%$ CI) & p-value ${ }^{a)}$ & HR $(95 \%$ CI) & p-value ${ }^{a)}$ \\
\hline Age $(\leq 65$ yr vs. $>65$ yr $)$ & $1.03(0.61-1.70)$ & 0.926 & & \\
\hline Sex (female vs. male) & $0.77(0.38-1.57)$ & 0.470 & & \\
\hline ECOG PS (0, 1 vs. 2$)$ & $0.60(0.34-1.05)$ & 0.072 & $0.64(0.36-1.16)$ & 0.140 \\
\hline Never vs. ever smoker & $1.81(0.71-4.60)$ & 0.212 & & \\
\hline Previous chemotherapy (1 vs. 2 ) & $0.98(0.59-1.63)$ & 0.945 & & \\
\hline EGFR (mutated vs. wild type) & $0.42(0.13-1.36)$ & 0.147 & & \\
\hline EGFR/FISH ${ }^{+}$vs. FISH $^{-}$ & $1.03(0.41-2.61)$ & 0.946 & & \\
\hline EGFR IHC $^{+}$vs. IHC ${ }^{-}$ & $0.99(0.56-1.75)$ & 0.977 & & \\
\hline Skin rash grade ( $\geq 2$ vs. $<2$ ) & $0.44(0.25-0.78)$ & 0.005 & $0.55(0.30-0.99)$ & 0.049 \\
\hline Stomatitis grade $(\geq 2$ vs. $<2)$ & $0.65(0.39-1.10)$ & 0.107 & & \\
\hline Diarrhea grade ( $\geq 2$ vs. $<2)$ & $0.98(0.59-1.63)$ & 0.946 & & \\
\hline
\end{tabular}

HR, hazard ratio; CI, confidential interval; ECOG, Eastern Cooperative Group; PS, performance status; FISH, fluorescence in

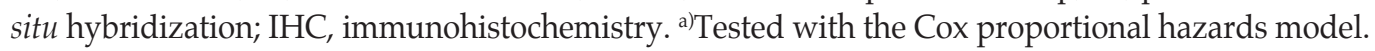

$\mathrm{p}=0.012$ ). Upon multivariate analysis of PFS, only the skin rash grade was significantly related to the risk of progression (HR for skin rash grade $\geq 2$ vs. grade $<2,0.44 ; 95 \% \mathrm{CI}, 0.25$ to $0.78 ; \mathrm{p}=0.005$ ) (Table 5). When analysis was limited to rashevaluable patients who continued treatment until at least 4 weeks, the difference in the progression of risk between groups remained unchanged (HR for skin rash grade $\geq 2$ vs. grade $<2,0.42 ; 95 \% \mathrm{CI}, 0.22$ to $0.81 ; \mathrm{p}=0.010$ ).

The dose reduction of afatinib was not significantly related to the ORR (yes vs. no, $14.3 \%$ vs. $5.6 \%$, $\mathrm{p}=0.575$ ). The risk of progression was the same in patients taking a reduced dose (HR for yes vs. no, 1.01; 95\% CI, 0.54 to $1.89 ; \mathrm{p}=0.967$ ). 


\section{Discussion}

To the best of our knowledge, this is the first clinical study to evaluate the emerging role of statins in overcoming resistance to EGFR-TKIs in patients with NA-NSCLC, which is usually resistant to EGFR-TKI therapy. The positive findings observed in the subgroup analysis of a previous clinical study were not confirmed in the present study [13]. The combination of AS in patients with NA-NSCLC did not improve the tumor response or survival outcomes compared with afatinib alone, although it did not increase the toxicities. These results were consistent with those observed for patients with tumors harboring WT EGFR.

Afatinib was recently approved for pretreated advanced NSCLC based on its superior efficacy compared with erlotinib in the LUX-Lung 8 trial [23]. The LUX-Lung 8 was a phase III randomized controlled trial that enrolled 795 pretreated patients with advanced squamous NSCLC globally to receive either erlotinib or afatinib, with a primary endpoint of PFS. The median PFS was significantly longer for afatinib than for erlotinib, (2.4 months vs. 1.9 months; HR, $0.82 ; \mathrm{p}=0.0427)$, as was the median OS (7.9 months vs. 6.8 months; HR, 0.81; $\mathrm{p}=0.0077)$. Although this global trial was the most recent positive study of patients with squamous cell lung carcinoma, the clinical benefits of the superior arm were also modest. Thus, the role of predictive markers in selecting patients who are likely to benefit more from EGFR-TKIs is more important in this lung cancer population. Our study searched for predictive markers for afatinib treatment in the entire study population with NA-NSCLC. There were no biomarkers related to the EGFR pathway that were predictive of RR and PFS for afatinib treatment. The EGFR mutation and EGFR/FISH status were significantly associated with RR, but not median PFS, whereas EGFR IHC status was not related to either RR or PFS. Interestingly, the most common adverse event for afatinib, skin rash, was significantly associated with risk of progression in this study. This finding was consistent with the landmark analysis at 4 weeks, which was measured because skin rash generally develops after 1 week and reaches maximum severity after 2 to 3 weeks of EGFRTKI treatment. Although several retrospective studies suggested that rash severity was associated with response or survival to EGFR-TKI treatment in NSCLC patients, a prospective study to evaluate the severity of skin rash as a surrogate marker for EGFR-TKI efficacy is needed [24-26]. Indeed, such a study will help guide EGFR-TKI treatment in NSCLC with WT EGFR, which lacks reliable predictive markers for EGFR-TKI efficacy.

The type, incidence, and severity of adverse effects in this study were comparable with those observed in another study with afatinib [23]. In the present study, grade $\geq 3$ skin rash, stomatitis, and diarrhea developed in $2.9 \%, 2.9 \%$, and $11.8 \%$ of patients, respectively, whereas they developed in $6.0 \%$, $4.0 \%$, and $10 \%$ of patients in a LUX-Lung 8 study [24]. In the present study, $26.6 \%$ of all patients reduced their afatinib dose, although the dose reduction did not affect the efficacy. Patients who reduced their afatinib dose to $30 \mathrm{mg}$ / day did not show inferior clinical outcomes compared with those who did not. These findings suggest that the afatinib dose can be adjusted in patients experiencing potentially challenging side effects.

Interestingly, one patient showed a CR to afatinib for a long duration, even though he had squamous cell carcinoma NSCLC. The histological type was squamous cell carcinoma in both surgical specimens of the primary tumor and right adrenal gland metastasis. An EGFR exon 19 deletion mutation was detected in his resected lung tumor sample. Currently, the role of the EGFR mutation test in lung squamous cell carcinoma remains controversial; thus, the guidelines for this test are not consistent among oncology societies. Although activating mutations are rare in lung squamous cell carcinoma $(<5 \%)$, the treatment outcome with EGFR-TKI in squamous cell carcinoma with the EGFR mutation is not inferior to that in adenocarcinoma with the EGFR mutation, as shown in the CR case in our study $[25,27]$. Thus, for personalized medicine with comprehensive genomic analysis, an EGFR mutation test also should be included to select the correct drugs for patients with lung squamous cell carcinoma.

There are several issues to consider in interpreting the negative results of this study. First, the median PFS of the A arm in the present study was longer than that of the afatinib arm in the LUX-Lung 8 trial (3.6 months vs. 2.4 months). Five patients (15.6\%) in the A arm withdrew from our study early; thus, the results may have been affected by this considerable number of censored events. In contrast, the median PFS of the AS arm in the present study was shorter than that of the afatinib arm in the LUX-8 trial (1.0 months vs. 2.4 months). This finding may be due to earlier assessment of the first tumor (4 weeks in this study vs. 8 weeks in the LUX-8 trial). Secondly, the dose of simvastatin used in this clinical study may have been too low to exert an anticancer effect when compared with the statin dose in preclinical studies. Despite the in vitro mechanistic rationale of statin studies, they have often been criticized for using high concentrations of statins (1-200 $\mu \mathrm{M})$ because the maximum statin concentrations in the serum of patients receiving standard doses for hyperlipidemia therapy are 10-200 $\mathrm{nM}[28,29]$. Whether the statins at higher doses confer therapeutic benefits to cancer patients has yet to be determined. Additionally, the resistance mechanism for EGFR-TKI of NA-NSCLC may be more complicated than RAS activation and downstream signaling cascades, which are expected to be inhibited by statins. In 
another phase II study of patients with KRAS mutant refractory colorectal tumors, adding simvastatin to cetuximab and irinotecan produced highly favorable clinical outcomes with a disease control rate of $65.4 \%$, a PFS of 7.6 months, and an OS of 12.8 months [30]. Thus, the combination of statins and EGFR-blocking agents may be effective in specific tumor types that are dependent on RAS and its downstream pathways.

The addition of simvastatin to afatinib did not improve efficacy versus afatinib alone in pretreated patients with NANSCLC. However, no unexpected adverse events were observed in response to treatment with combined AS. EGFR mutation, EGFR FISH, and EGFR IHS status were not reliable as predictive markers for afatinib efficacy. However, skin rash severity may be useful for making treatment decisions regarding afatinib in this population.

\section{Conflicts of Interest}

The study was partly supported by Boehringer Ingelheim.

\section{Acknowledgments}

The study was partly supported by a National Cancer Center Research grant (14100660).

\section{References}

1. Gazdar AF. Epidermal growth factor receptor inhibition in lung cancer: the evolving role of individualized therapy. Cancer Metastasis Rev. 2010;29:37-48.

2. Lynch TJ, Bell DW, Sordella R, Gurubhagavatula S, Okimoto RA, Brannigan BW, et al. Activating mutations in the epidermal growth factor receptor underlying responsiveness of nonsmall-cell lung cancer to gefitinib. N Engl J Med. 2004;350: 2129-39.

3. Paez JG, Janne PA, Lee JC, Tracy S, Greulich H, Gabriel S, et al. EGFR mutations in lung cancer: correlation with clinical response to gefitinib therapy. Science. 2004;304:1497-500.

4. Mitsudomi T, Morita S, Yatabe Y, Negoro S, Okamoto I, Tsurutani J, et al. Gefitinib versus cisplatin plus docetaxel in patients with non-small-cell lung cancer harbouring mutations of the epidermal growth factor receptor (WJTOG3405): an open label, randomised phase 3 trial. Lancet Oncol. 2010;11: 121-8.

5. Maemondo M, Inoue A, Kobayashi K, Sugawara S, Oizumi S, Isobe $\mathrm{H}$, et al. Gefitinib or chemotherapy for non-small-cell lung cancer with mutated EGFR. N Engl J Med. 2010;362: 2380-8.

6. Rosell R, Carcereny E, Gervais R, Vergnenegre A, Massuti B, Felip E, et al. Erlotinib versus standard chemotherapy as firstline treatment for European patients with advanced EGFR mutation-positive non-small-cell lung cancer (EURTAC): a multicentre, open-label, randomised phase 3 trial. Lancet Oncol. 2012;13:239-46.

7. Zhou C, Wu YL, Chen G, Feng J, Liu XQ, Wang C, et al. Erlotinib versus chemotherapy as first-line treatment for patients with advanced EGFR mutation-positive non-smallcell lung cancer (OPTIMAL, CTONG-0802): a multicentre, open-label, randomised, phase 3 study. Lancet Oncol. 2011;12: 735-42.

8. Wu YL, Zhou C, Hu CP, Feng J, Lu S, Huang Y, et al. Afatinib versus cisplatin plus gemcitabine for first-line treatment of
Asian patients with advanced non-small-cell lung cancer harbouring EGFR mutations (LUX-Lung 6): an open-label, randomised phase 3 trial. Lancet Oncol. 2014;15:213-22.

9. Lee JK, Hahn S, Kim DW, Suh KJ, Keam B, Kim TM, et al. Epidermal growth factor receptor tyrosine kinase inhibitors vs conventional chemotherapy in non-small cell lung cancer harboring wild-type epidermal growth factor receptor: a metaanalysis. JAMA. 2014;311:1430-7.

10. Shepherd FA, Rodrigues Pereira J, Ciuleanu T, Tan EH, Hirsh $\mathrm{V}$, Thongprasert $\mathrm{S}$, et al. Erlotinib in previously treated nonsmall-cell lung cancer. N Engl J Med. 2005;353:123-32.

11. Han JY, Lee SH, Yoo NJ, Hyung LS, Moon YJ, Yun T, et al. A randomized phase II study of gefitinib plus simvastatin versus gefitinib alone in previously treated patients with advanced non-small cell lung cancer. Clin Cancer Res. 2011;17:1553-60.

12. Cortot AB, Janne PA. Molecular mechanisms of resistance in epidermal growth factor receptor-mutant lung adenocarcinomas. Eur Respir Rev. 2014;23:356-66.

13. Endo A. The discovery and development of HMG-CoA reductase inhibitors. 1992. Atheroscler Suppl. 2004;5:67-80.

14. Seabra MC, Mules EH, Hume AN. Rab GTPases, intracellular traffic and disease. Trends Mol Med. 2002;8:23-30.

15. Pruitt K, Der CJ. Ras and Rho regulation of the cell cycle and oncogenesis. Cancer Lett. 2001;171:1-10.

16. Mantha AJ, Hanson JE, Goss G, Lagarde AE, Lorimer IA, Dimitroulakos J. Targeting the mevalonate pathway inhibits the function of the epidermal growth factor receptor. Clin Cancer Res. 2005;11:2398-407.

17. Park IH, Kim JY, Jung JI, Han JY. Lovastatin overcomes gefitinib resistance in human non-small cell lung cancer cells with K-Ras mutations. Invest New Drugs. 2010;28:791-9.

18. Prueksaritanont T, Ma B, Yu N. The human hepatic metabolism of simvastatin hydroxy acid is mediated primarily by CYP3A, and not CYP2D6. Br J Clin Pharmacol. 2003;56:120-4. 19. Peters S, Zimmermann S, Adjei AA. Oral epidermal growth 
factor receptor tyrosine kinase inhibitors for the treatment of non-small cell lung cancer: comparative pharmacokinetics and drug-drug interactions. Cancer Treat Rev. 2014;40:917-26.

20. Eisenhauer EA, Therasse P, Bogaerts J, Schwartz LH, Sargent D, Ford R, et al. New response evaluation criteria in solid tumours: revised RECIST guideline (version 1.1). Eur J Cancer. 2009;45:228-47.

21. Lee DH, Lee GK, Kong SY, Kook MC, Yang SK, Park SY, et al. Epidermal growth factor receptor status in anaplastic thyroid carcinoma. J Clin Pathol. 2007;60:881-4.

22. Hirsch FR, Varella-Garcia M, Bunn PA Jr, Di Maria MV, Veve $\mathrm{R}$, Bremmes RM, et al. Epidermal growth factor receptor in non-small-cell lung carcinomas: correlation between gene copy number and protein expression and impact on prognosis. J Clin Oncol. 2003;21:3798-807.

23. Soria JC, Felip E, Cobo M, Lu S, Syrigos K, Lee KH, et al. Afatinib versus erlotinib as second-line treatment of patients with advanced squamous cell carcinoma of the lung (LUX-Lung 8): an open-label randomised controlled phase 3 trial. Lancet Oncol. 2015;16:897-907.

24. Kudo K, Hotta K, Bessho A, Nogami N, Kozuki T, Kuyama S, et al. Development of a skin rash within the first week and the therapeutic effect in afatinib monotherapy for EGFR-mutant non-small cell lung cancer (NSCLC): Okayama Lung Cancer Study Group experience. Cancer Chemother Pharmacol.
2016;77:1005-9.

25. Lee Y, Shim HS, Park MS, Kim JH, Ha SJ, Kim SH, et al. High EGFR gene copy number and skin rash as predictive markers for EGFR tyrosine kinase inhibitors in patients with advanced squamous cell lung carcinoma. Clin Cancer Res. 2012;18: 1760-8.

26. Hata A, Katakami N, Kunimasa K, Yoshioka H, Fujita S, Kaji $\mathrm{R}$, et al. Erlotinib for pretreated squamous cell carcinoma of the lung in Japanese patients. Jpn J Clin Oncol. 2011;41: 1366-72.

27. Cappuzzo F, Hirsch FR, Rossi E, Bartolini S, Ceresoli GL, Bemis L, et al. Epidermal growth factor receptor gene and protein and gefitinib sensitivity in non-small-cell lung cancer. J Natl Cancer Inst. 2005;97:643-55.

28. Wong WW, Dimitroulakos J, Minden MD, Penn LZ. HMGCoA reductase inhibitors and the malignant cell: the statin family of drugs as triggers of tumor-specific apoptosis. Leukemia. 2002;16:508-19.

29. Demierre MF, Higgins PD, Gruber SB, Hawk E, Lippman SM. Statins and cancer prevention. Nat Rev Cancer. 2005;5:930-42.

30. Lee J, Hong YS, Hong JY, Han SW, Kim TW, Kang HJ, et al. Effect of simvastatin plus cetuximab/irinotecan for KRAS mutant colorectal cancer and predictive value of the RAS signature for treatment response to cetuximab. Invest New Drugs. 2014;32:535-41. 